\title{
Design of Remote Wireless Monitoring System of Substations Based on GPRS
}

\author{
Wang Xiaohui ${ }^{1}$, Wu Chunming ${ }^{2}$ and Wang Nannan ${ }^{3}$ \\ ${ }^{1}$ Foreign Language School of Northeast Dianli University, JiLin China \\ ${ }^{2}$ School of Information Engineering of Northeast Dianli University, JiLin China \\ ${ }^{3}$ School of Automation Engineering of Northeast Dianli University, JiLin China \\ awxhxuna@126.com, b466389144@qq.com, ${ }^{\mathrm{c}} 287111782 @ q q . c o m$
}

Keywords: GPRS; substation; ARM; Monitoring

\begin{abstract}
As a place for voltage and current to change, focus and allocate, substation is an important node in the power system. The safe operation of its equipments plays a critical role in the safe and stable operation of the power system. The automation and unattended situation of substations is currently two hot spots in the field of smart power grids. According to the current technology in the field of electric power remote monitoring system for reference, we proposed and designed a wireless, real-time, multi-object remote monitoring system for electric power equipment. The system has fast transmission rates, low cost and can directly interconnect with the Internet. It can run on parameters of the power equipment in the substation in real-time remote monitoring.
\end{abstract}

\section{Introduction}

With the rapid improvement of peoples' living standard humans depend on electricity more and more. How to keep the electric system stable is of significance for peoples' happy life besides increasing power generation capacity. It plays the key role in stable and safe operation of the whole electric power that the various critical nodes in electric power run smoothly, such as voltage, current, power frequency, etc. Therefore, it is very significant to monitor them. Substations undertake the main task to transmit electric power and they require real-time monitoring in order to get a reliable and economical electric power.

Hereby, we will design the hardware of on-site terminal by using S3C6410X(Microprocessor) ${ }^{[1]}$ from Samsung as the main controller and SIM300 ${ }^{[2]}$ from SIMCOM as the GPRS communication module, whose advantages lie in the promotion of running velocity for the system and strengthening data processing capacity.

\section{The general design of the system}

The system mainly includes data collection on-site terminal, data wireless transmitting network and monitoring center in the substations. 6 important parameters are selected as supervising objects, which are transmitted to ADC connector after the collection and dispatched to the monitoring center through GPRS modules, GPRS net and Internet ${ }^{[3]}$. The general block diagrams of system are shown in diagram 1. 


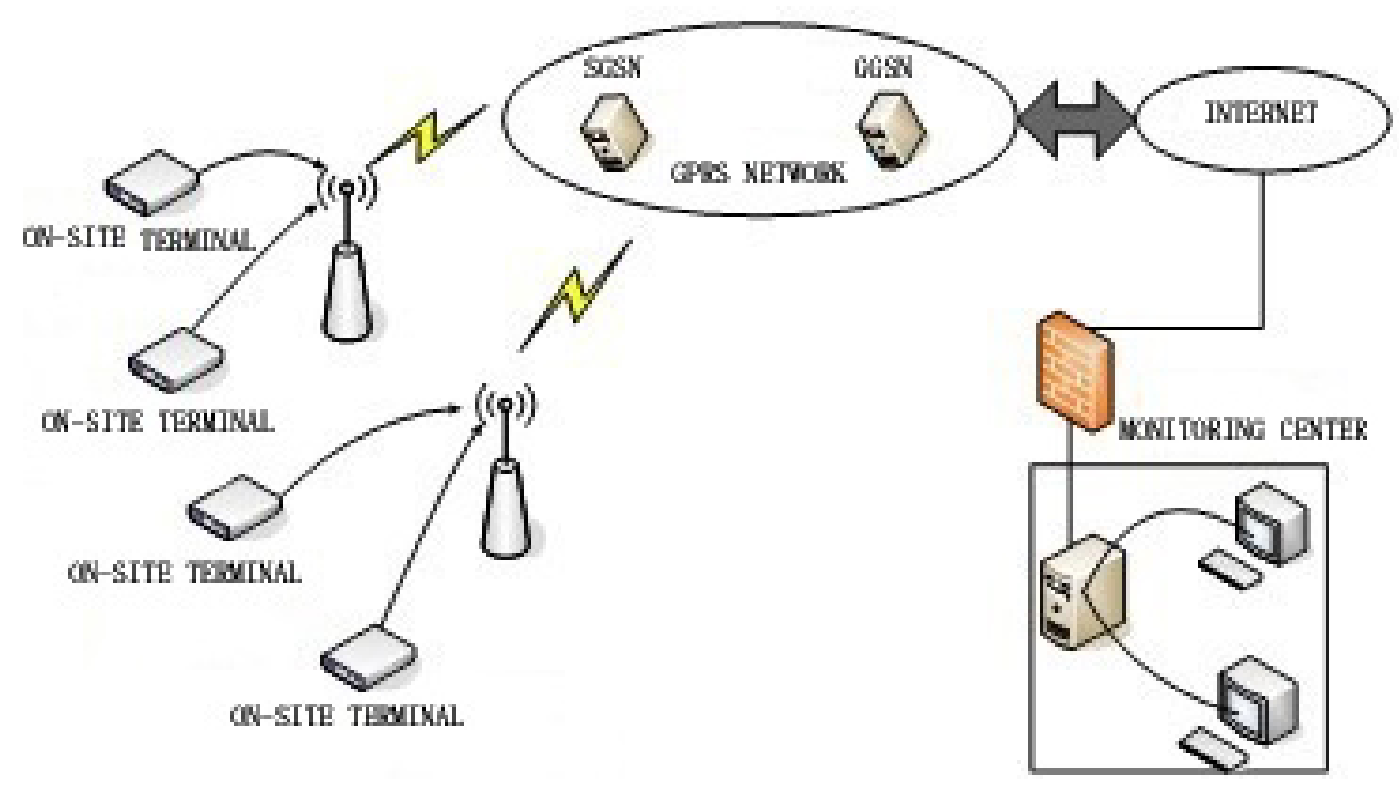

Fig :1 block diagram of the system

\section{Hardware Design of On-Site terminal}

The hardware of on-site terminal in substations should meet the needs of real-time monitoring electric equipments, core devices of which include ARM microprocessors and GPRS communication modules as well as some necessary peripheral circuits, for example, storage, JTAG, LCD,RS232 and system power. The following is the structure chart of real-time terminal hardware.

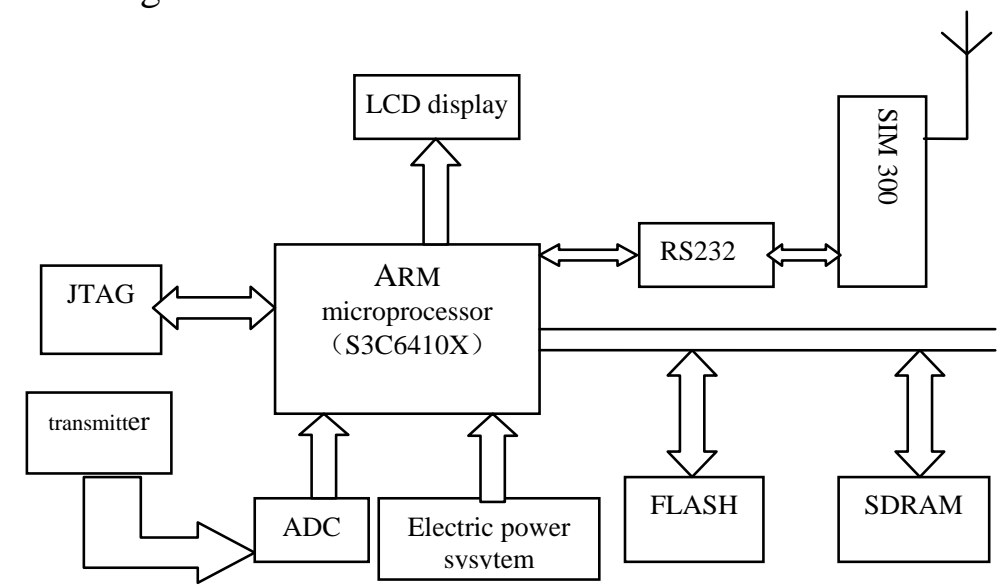

Fig 2: Hardware structure chart of on-site terminal

Design of expander circuit of storage.Storage space is where embedded system conserves its routine and data in application and it serves as a space which provides the according management to S3C6410X when microprocessor chips need. As is well known, there are many temporary storages inside S3C6410X chips which store many important information and accelerate the running velocity of microprocessors. But it is far from enough if we only use these temporary storages as an embedded applicable system in that a large number of application programs and data need conserving. As a result, storage space needs to extended in the external part of S3C6410X chips. The fact that address decoding circuit is integrated inside S3C6410X chips(the controller of storage space expansion) reduces peripheral circuit, which makes it easier to expand storage space. 


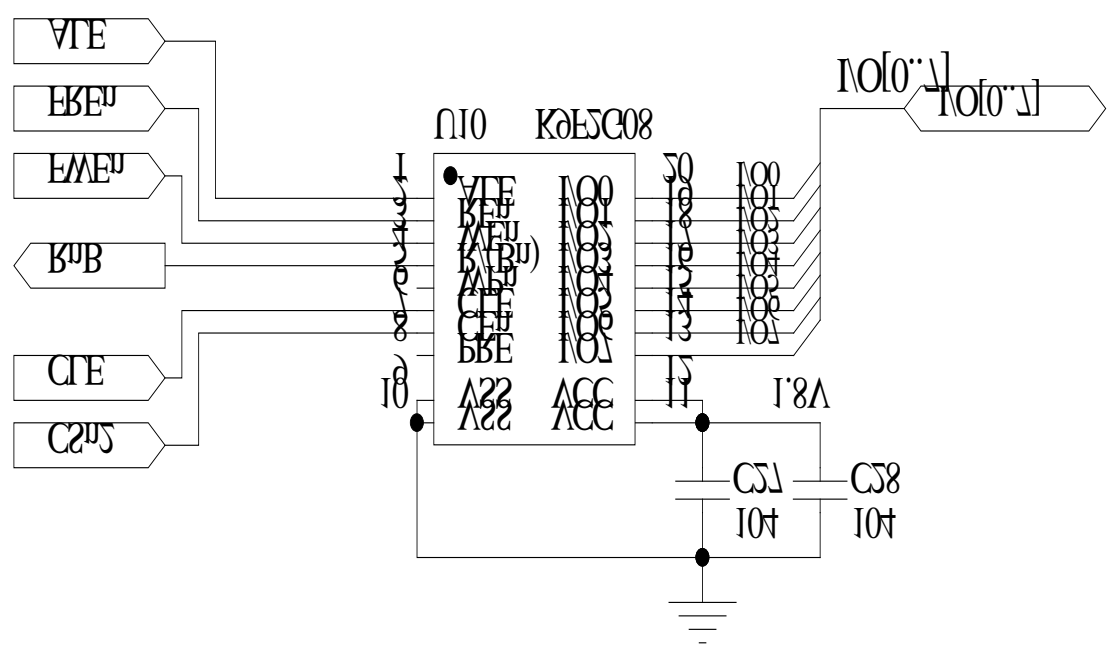

Fig 3: K9F2G08 Expansive circuit diagram

According to the above features, when we use Samsung's NAND Flash chips K9F2G08 ${ }^{[4]}$ and Mobile DDR RAM chips K4X51163P ${ }^{[5]}$, the capacity is respectively 256MB and 32MB. Because K4X51163P chips only have 16 digits data bus, 2 chips can meet the requirements of S3C6410X's 32 digits data bus during the expansion. Circuit diagram of memory expansion is as shown in diagram 3.

Design of the system's on-site parameters acquisition circuit.Such parameters as busbar voltage, active power and reactive power, etc, can't be collected directly in substations and some auxiliary equipment are needed to finish it. Voltage and current which are decreased by voltage and current transformer are sent into microprocessor S3C6410X where S3C6410X has analog-digital converter(ADC) which has 8 signal input channel. Users can choose to collect any one of the signal data by installing a simulated multiple-way switch. The system adopts its 6 simulated input channels and its diagram of parameters collection is shown as the following.

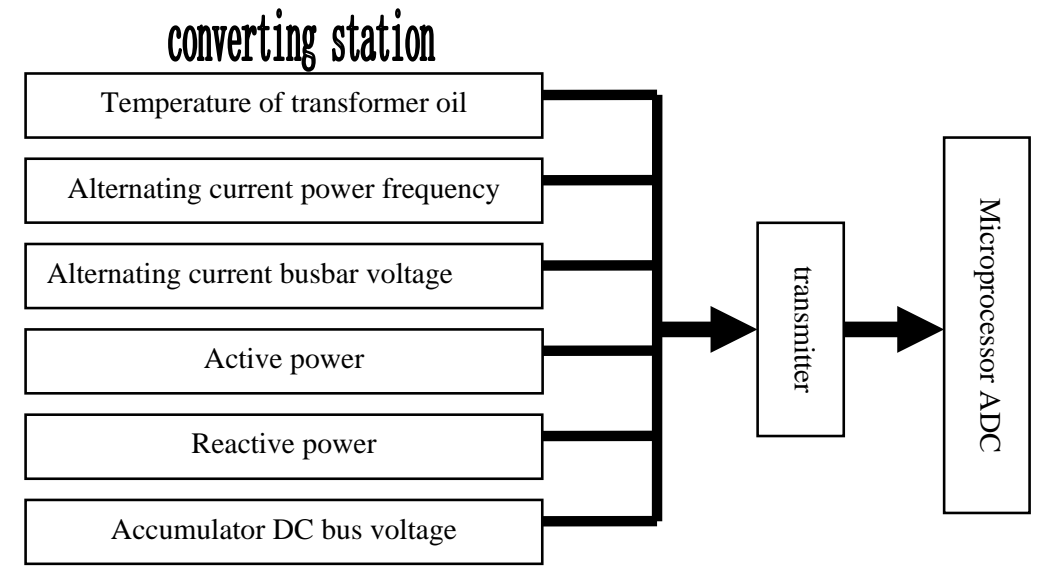

Fig 4: schematic diagram of parameters collection

\section{Software design of on-site terminal}

The part of software design focuses on the transplantation of real-time operating system (Linux) onto S3C6410X. The main program tree is drawn based on knowing Linux operating system which develops very fast and is used widely and is famous for high efficiency and flexibility ${ }^{[6]}$. Linux has many advantages. Firstly, it can operate on many platforms. Besides, its source code is open and it's free. Finally, it abides by GPL spirit and POSIX standard as well as its compatibility with Unix. Hence, it's a real operating system of multi-user and multi-task and it can provide interpretation program of shell commands and programming language.

According to the above advantages and special features and taking consideration of the requirements of embedded operating system, it is feasible to transplant Linux real-time operating 
system onto the microprocessor S3C6410X. In theory the main program tree is displayed as the following.

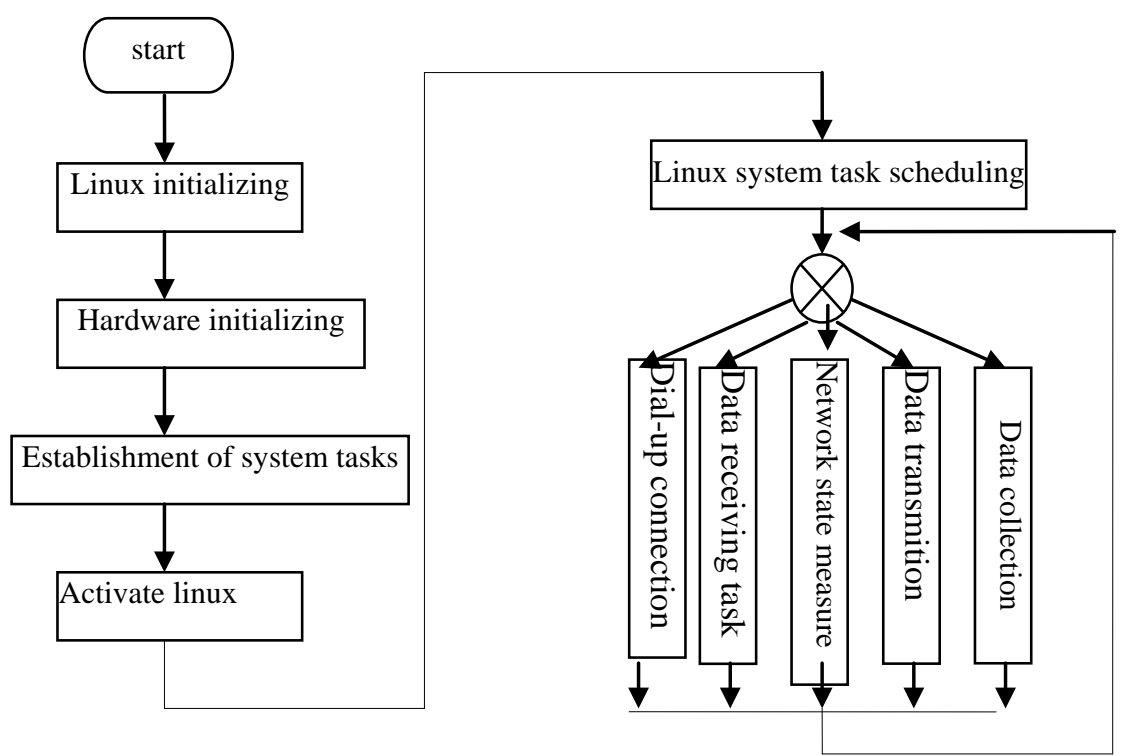

Fig 5: program flow chart of main program

\section{Summary}

The core of the on-site terminal of substations is based on 32 digital embedded ARM processor S3C6410X from Samsung and communication modules SIM300 from SIMCOM. The hardware system of on-site terminal is built through expanding external storage and interface circuit such as serial port. The software part can be realized under the linux embedded real-time operating system which can be divided into many tasks such as data collection. This design almost makes the expectable function of on-site terminal come true and realizes the communication between on-site terminal and monitoring center.

\section{References}

[1] Samsung Electronics.S3C6410X_UM_Rev1.10 Data Sheet,2008.

[2] Simcom.sim300 spec.

[3] Huaiyun,Nie, Research and Design of remote monitoring system of shipping based on $\mu \mathrm{C} / \mathrm{OS}$ II and GPRS, Nanjing University of science and technology masters' thesis, 2006

[4] Samsung Electronics.K9F2G08Q0M Data Sheet,2004.

[5] Samsung Electronics.K4X51163PC Data Sheet,2006.

[6] Kaiyu, Zhang, Lixin, Song, Design of data transmission terminal cell based on GPS, [J]. Journal of Harbin university of science and technology, 2007,12(6):29 